\title{
An Approach to Hybrid Optimal Smoothing for Linear Discrete-Time Systems with Non-Gaussian Noises
}

\author{
Gou Nakura
}

E-mail: gg9925_fiesta@ybb.ne.jp

\begin{abstract}
In this paper we study hybrid estimation for linear discrete-time systems with noises not to be restricted to be Gaussian. It is assumed that modes of the systems are not directly accessible. We consider optimal estimation problems to find both estimated states of the systems and a candidate of the distributions of the modes over the finite time interval. We adopt most probable trajectory (MPT) approach. Q. Zhang (1999, 2000) has presented hybrid filtering algorithm, i.e., causal estimation, by MPT approach for linear continuous- and discrete-time hybrid systems with non-Gaussian noises. We consider both filtering and smoothing problems for the linear discrete-time hybrid systems in this paper. Based on the principles of hybrid optimality we present filtering and smoothing algorithms, which give the solutions of these estimation problems. In the smoothing case, we can expect better estimation performance by taking into consideration noncausal information of observations. The hybrid smoother is realized by two filters approach ([23]).
\end{abstract}

Key Words: Hybrid systems; Noncausal estimation; Non-Gaussian noise; Two filters approach; Smoothing

\section{Introduction}

It is very important to consider simultaneous estimation of both system states and inaccessible modes for hybrid systems with unknown modes $([4,7,24,25])$. This estimation is called hybrid estimation. By the hybrid estimation we often want to know both a current mode and system state at each time through information of observation. However there exist cases that we want to know distributions of modes on long run time interval rather than each estimate of the modes themselves at each time to grasp global performance over long time intervals, for example, distributions of active modes in solar systems $([5,21])$, distributions of active agents on formation or consensus via hybrid systems representation over long run time and so on.

Much work has been done for smoothing theory for both of continuous- and discrete-time systems ([1, 2, 3, $6,8,9,10,12,13,14,15,18,19,20,22,23]$ and so on). Various researchers have studied the smoothing problems by various approach, for example, maximum likeli- hood approach $([9,13,19])$, projection approach $([14])$ and so on. In the meaning of utilization of accumulated information of observation until current time and estimation of state on fixed-time interval smoothing is noncausal estimation. It is well known that smoothers (noncausal estimators) more effectively estimates the states than filters (causal estimators) because of more information of observation. Nevertheless, on research of estimation for hybrid systems, little work has been done from the point of view of the noncausal information of observation, i.e., smoothing. In [9] Helmick et al. have presented a fixed-interval smoothing algorithm for discrete-time Markovian jump systems by maximum likelihood (ML) approach. However they have considered only the case with fully accessible modes and their approach is based on approximate approach to probability density functions (PDFs). Therefore they have presented only a nearly optimal smoothing algorithm. While it is significant that optimality is guaranteed for estimation algorithms, in [4] and [7] Costa et al. have presented LMMSE (linear minimum mean square estimate) filters to estimate both system states and inaccessible modes for continuous- and discretetime Markovian jump systems affected by wide sense white noises, but in these LMMSE filters theory the optimality of estimation isn't always guaranteed in the meaning that these filters aren't always MMSE (minimum mean square estimate). To the best of the author's knowledge the optimal smoothing problems in the cases with inaccessible modes have not yet fully investigated.

In this paper we study hybrid estimation for linear discrete-time systems with non-Gaussian noises. The concerned systems are general hybrid systems given below which aren't restricted to Markovian jump systems $([4,5,7])$ and where added noises aren't restricted to be Gaussian. It is assumed that modes of the systems are not directly accessible throughout this paper. We consider optimal estimation problems to find both estimated states of the systems and an optimal candidate of the distributions of the modes over the finite time interval. We adopt most probable trajectory (MPT) approach to guarantee the optimality of estimation methods. On this approach, given information of observation, we consider optimal control problems where we seek optimal control by which averaged noises 
energies are minimized for averaged systems throughout the mode distributions. In [24, 25] Zhang has presented hybrid filtering algorithm by MPT approach for the continuous- and discrete-time hybrid systems. We consider both filtering and smoothing problems for discrete-time hybrid systems in this paper. We show that principles of optimality hold for the concerned systems and performance indices and, based on the principles, construct the hybrid estimation algorithms. In the smoothing case we can expect better estimation performance by taking into consideration noncausal information of observations. The hybrid smoother is realized by two filters approach $([2,8,12,20,22,23])$. Finally we give numerical examples and verify that we can obtain better estimation performance by smoothing than filtering.

\section{Systems and Problems Formulation}

Let $(\Omega, \mathcal{F}, \mathcal{P})$ be a probability space and, on this space, we consider the following system with mode transitions and noises which aren't restricted to be Gaussian.

$$
\begin{gathered}
x(k+1)=A_{d}(k, \theta(k)) x(k)+w_{d}(k, \theta(k)), \\
x(0)=x_{0}, \theta(0)=i_{0} \\
y(k)=H_{d}(k, \theta(k)) x(k)+v_{d}(k, \theta(k))
\end{gathered}
$$

where $x \in \mathbf{R}^{\mathbf{n}}$ is the state, $w_{d} \in \mathbf{R}^{\mathbf{n}}$ is the exogenous random noise, $v_{d} \in \mathbf{R}^{\mathbf{k}}$ is the measurement noise, and $y \in \mathbf{R}^{\mathbf{k}}$ is the measured output. $x_{0}$ is an unknown initial state and it is assumed that a distribution of initial modes $i_{0}$ is given. The noises $w_{d}(\cdot, \cdot)$ and $v_{d}(\cdot, \cdot)$ aren't restricted to be Gaussian.

We assume that all these matrices are of compatible dimensions.

Let $\mathcal{M}=\{1,2, \cdots, m\}$ denote the state space of $\theta(k)$. In this paper it is assumed that the probability distribution of $\theta(\cdot)$ is unknown, but among a finite number of candidate distributions. Let $r \in \mathcal{N}_{0}=\left\{1,2, \cdots, n_{0}\right\}$, and let $\mathcal{P}=\left\{\phi^{(1)}(\cdot), \cdots, \phi^{\left(n_{0}\right)}(\cdot)\right\}$ denote the set of such candidate distributions on $\mathcal{M}$, i.e., for $r \in \mathcal{N}_{0}$ and $k \in[0, N], \phi^{(r)}(k)=\left(\phi_{1}^{(r)}(k), \cdots, \phi_{m}^{(r)}(k)\right)$ with $\phi_{i}^{(r)}(k) \geq 0$ and $\sum_{i=1}^{m} \phi_{i}^{(r)}(k)=1$.

The fixed-interval smoothing problem we address in this paper for the system (1) is to find the MPT (most probable trajectory) estimate of $x(k), k \in[0, N]$, over the finite horizon $[0, N]$, using the information available on the known part of the observation $y(\cdot)$ for the given distributions of initial mode $i_{0}$ and initial state $x_{0}$. We define the following performance indices for $r \in \mathcal{N}_{0}$ and $k \in[0, N]:$

$$
\begin{aligned}
& J_{0 k}^{(r)}\left(x_{0}, w_{d}, v_{d}\right) \\
:= & \sum_{l=0}^{k-1} \sum_{i=1}^{m} \phi_{i}^{(r)}(l)\left(w_{d}^{\prime}(l, i) M_{d}(l, i) w_{d}(l, i)\right.
\end{aligned}
$$

$$
\begin{gathered}
\left.+v_{d}^{\prime}(l, i) N_{d}(l, i) v_{d}(l, i)\right) \\
+\left(x_{0}-\hat{x}_{0}\right)^{\prime} D_{0}\left(x_{0}-\hat{x}(0)\right) \\
J_{0 N}^{(r)}\left(x_{0}, w_{d}, v_{d}\right) \\
:=\sum_{l=0}^{N-1} \sum_{i=1}^{m} \phi_{i}^{(r)}(l)\left(w_{d}^{\prime}(l, i) M_{d}(l, i) w_{d}(l, i)\right. \\
\left.+v_{d}^{\prime}(l, i) N_{d}(l, i) v_{d}(l, i)\right) \\
+\left(x_{0}-\hat{x}_{0}\right)^{\prime} D_{0}\left(x_{0}-\hat{x}(0)\right) \\
\quad+\left(x(N)-\hat{x}_{N}\right)^{\prime} D_{N}\left(x(N)-\hat{x}_{N}\right)
\end{gathered}
$$

where $\hat{x}_{0}$ is an initial estimate of $x_{0}$ and $\hat{x}_{N}$ is a terminal estimate of $x(N) . M_{d}(l, i)>O, N_{d}(l, i) \geq O$, $D_{0}>O$ and $D_{N}>O$ are symmetric matrices which reflect the uncertainties on the noises $w_{d}(\cdot, \cdot), v_{d}(\cdot, \cdot)$, the estimates $\hat{x}_{0}$ and $\hat{x}_{N}$. Thus these performance indices mean the energies of noises, initial and terminal estimates under some uncertainties averaged by the mode distributions for each $r \in \mathcal{N}_{0}$. We consider the optimization problems to decide $w_{d}(\cdot, i), v_{d}(\cdot, i)$ and $r \in \mathcal{N}_{0}$ minimizing $J_{0 k}^{(r)}$ and $J_{0 N}^{(r)}$ utilizing the known parts of the observed information $\mathcal{Y}_{N}=\{y(l) \mid 0 \leq l \leq N\}$.

Since the mode $\theta(k)$ at each time is inaccessible, we cannot directly design estimators for the system (1) including the unknown modes. Also, even if the modes are accessible, the computational complexity can exponentially increase if we directly design the estimators for the system (1) including $\theta(k)$ explicitly. Hence we introduce the system averaged through the mode distributions for each $r \in \mathcal{N}_{0}$.

For notational simplicity, we adopt the following notation.

$$
\bar{F}^{(r)}(k)=\sum_{i=1}^{m} \phi_{i}^{(r)}(k) F(k, i)
$$

for a matrix function $F(k, i)$ and $r \in \mathcal{N}_{0}$. Similarly ${\overline{F_{1} F_{2}}}^{(r)}(k)=\sum_{i=1}^{m} \phi_{i}^{(r)}(k) F_{1}(k, i) F_{2}(k, i)$ for matrix functions $F_{1}(k, i)$ and $F_{2}(k, i)$ and so on. Using these notations, we can shift the drift term in the system (1) to $\bar{A}^{(r)}(k)$ as follows:

$$
x(k+1)={\overline{A_{d}}}^{(r)}(k) x(k)+w_{d}(k)
$$

where

$$
\begin{aligned}
& w_{d}(k)=w_{d}^{(r)}(k) \\
= & \left(A_{d}(k, \theta(k))-{\overline{A_{d}}}^{(r)}(k)\right) x(k)+w_{d}(k, \theta(k)) .
\end{aligned}
$$

By replacing the system noise $w_{d}(k, i)$ by ${\overline{A_{d}}}^{(r)}(k)-$ $\left.A_{d}(k, i)\right) x(k)+w_{d}(k)$ and the observation noise $v_{d}(k, i)$ by $y(k)-H_{d}(k, i) x(k)$ in the performance indices $(2)$ and (3), we define

$$
\begin{aligned}
& L^{(r)}\left(k, x, w_{d}, y\right) \\
& :=\sum_{i=1}^{m} \phi_{i}^{(r)}(k)\left(\left[{\overline{A_{d}}}^{(r)}(k)-A_{d}(k, i)\right) x+w_{d}\right]^{\prime} \\
& \times M_{d}(k, i)\left[\left({\overline{A_{d}}}^{(r)}(k)-A_{d}(k, i)\right) x+w_{d}\right] \\
& \left.+\left(y-H_{d}(k, i) x\right)^{\prime} N_{d}(k, i)\left(y-H_{d}(k, i) x\right)\right) .
\end{aligned}
$$


Then we can define the following performance indices:

$$
\begin{aligned}
& J_{f}^{(r)}\left(k, x, w_{d}(\cdot)\right) \\
:= & \sum_{l=0}^{k-1} L^{(r)}\left(l, x(l), w_{d}(l), y(l)\right)+\Phi_{0}(x(0)) \\
& J_{b}^{(r)}\left(k, x, w_{d}(\cdot)\right) \\
:= & \sum_{l=k}^{N-1} L^{(r)}\left(l, x(l), w_{d}(l), y(l)\right)+\Phi_{N}(x(N)) \\
& J_{s}^{(r)}\left(k, x, w_{d}(\cdot)\right) \\
:= & J_{f}^{(r)}\left(k, x, w_{d}(\cdot)\right)+J_{b}^{(r)}\left(k, x, w_{d}(\cdot)\right)
\end{aligned}
$$

where $\Phi_{0}(x(\cdot))=\left(x(\cdot)-\hat{x}_{0}\right)^{\prime} D_{0}\left(x(\cdot)-\hat{x}_{0}\right)$ and $\Phi_{N}(x(\cdot))=\left(x(\cdot)-\hat{x}_{N}\right)^{\prime} D_{N}\left(x(\cdot)-\hat{x}_{N}\right)$.

We consider the optimal control problems to minimize $J_{f}^{(r)}$ and $J_{s}^{(r)}=J_{f}^{(r)}+J_{b}^{(r)}$ for the given parts of $\mathcal{Y}_{N}$. Let $V_{f}^{(r)}(k, x)$ and $V_{b}^{(r)}(k, x)$ be the value functions of these control problems as follows:

$$
\begin{aligned}
V_{f}^{(r)}(k, x) & :=\inf _{w_{d}(\cdot)} J_{f}^{(r)}\left(k, x, w_{d}(\cdot)\right) \\
V_{b}^{(r)}(k, x) & :=\inf _{w_{d}(\cdot)} J_{b}^{(r)}\left(k, x, w_{d}(\cdot)\right) \\
V_{s}^{(r)}(k, x) & :=V_{f}^{(r)}(k, x)+V_{b}^{(r)}(k, x) \\
w_{d, f}^{(r) *}(k) & :=\arg \min \left\{J_{f}^{(r)}\left(k, x, w_{d}(k)\right): w \in \mathbf{R}^{\mathbf{n}}\right\} \\
w_{d, b}^{(r) *}(k) & :=\arg \min \left\{J_{b}^{(r)}\left(k, x, w_{d}(k)\right): w \in \mathbf{R}^{\mathbf{n}}\right\} \\
w_{d, s}^{(r) *}(k) & :=\arg \min \left\{J_{s}^{(r)}\left(k, x, w_{d}(k)\right): w \in \mathbf{R}^{\mathbf{n}}\right\}
\end{aligned}
$$

Then define

$$
\begin{aligned}
& \hat{x}_{f}^{(r)}(k):=\arg \min \left\{V_{f}^{(r)}(k, x): x \in \mathbf{R}^{\mathbf{n}}\right\}, \\
& V_{f}^{(r)}(k):=V_{f}^{(r)}\left(k, \hat{x}_{f}^{(r)}(k)\right)
\end{aligned}
$$

and

$$
\hat{r}_{f}(k):=\arg \min \left\{V_{f}^{(r)}(k): r \in \mathcal{N}_{0}\right\} .
$$

Then the most probable distribution is $\phi^{\left(\hat{r}_{f}(k)\right)}(\cdot)$. Let $\hat{x}_{f}(k)=\hat{x}_{f}^{\left(\hat{r}_{f}(k)\right)}(k)$ and we have

$$
\begin{aligned}
& V_{f}^{\left(\hat{r}_{f}(k)\right)}\left(k, \hat{x}_{f}(k)\right) \leq V_{f}^{(r)}\left(k, \hat{x}_{f}^{(r)}(k)\right) \\
\leq & V_{f}^{(r)}(k, x)=J_{f}^{(r)}\left(k, x, w_{d, f}^{(r) *}(k)\right) \leq J_{f}^{(r)}\left(k, x, w_{d}(k)\right) .
\end{aligned}
$$

Also define

$$
\begin{aligned}
& \hat{x}_{s}^{(r)}(k):=\arg \min \left\{V_{s}^{(r)}(k, x): x \in \mathbf{R}^{\mathbf{n}}\right\}, \\
& V_{s}^{(r)}(k):=V_{s}^{(r)}\left(k, \hat{x}_{s}^{(r)}(k)\right)
\end{aligned}
$$

and

$$
\hat{r}_{s}(k):=\arg \min \left\{V_{s}^{(r)}(k): r \in \mathcal{N}_{0}\right\} .
$$

Then the most probable distribution is $\phi^{\left(\hat{r}_{s}(k)\right)}(\cdot)$. Let $\hat{x}_{s}(k)=\hat{x}_{s}^{\left(\hat{r}_{s}(k)\right)}(k)$ and we have

$$
\begin{aligned}
& V_{s}^{\left(\hat{r}_{s}(k)\right)}\left(k, \hat{x}_{s}(k)\right) \leq V_{s}^{(r)}\left(k, \hat{x}_{s}^{(r)}(k)\right) \\
\leq & V_{s}^{(r)}(k, x)=J_{s}^{(r)}\left(k, x, w_{d, s}^{(r) *}(k)\right) \leq J_{s}^{(r)}\left(k, x, w_{d}(k)\right) .
\end{aligned}
$$

Now we define the following optimal estimators in the sense of most probable trajectories (MPTs).

Definition 2.1 Given the matrices $M_{d}, N_{d}, D_{0}$ and $D_{N},\left(\hat{r}_{f}(k), \hat{x}_{f}(k)\right), k \geq 0$, is called an optimal filter (in the MPT sense) if it minimizes $V_{f}^{(r)}(k, x)$. $\left(\hat{r}_{s}(k), \hat{x}_{s}(k)\right), 0 \leq k \leq N$ is called an optimal smoother (in the MPT sense) if it minimizes $V_{s}^{(r)}(k, x)$.

Then we formulate the following optimal hybrid estimation problems for the performance indices (4) and (6).

The Optimal Hybrid Filtering Problem for Linear Discrete-Time Systems:

Find the pair $\left(\hat{r}_{f}(l), \hat{x}_{f}^{\left(\hat{r}_{f}(l)\right)}(l)\right), l \in[0, k]$ minimizing the performance index (4) based on the causal part $\mathcal{Y}_{k}=\{y(l) \mid 0 \leq l \leq k\}$ of the observed information $\mathcal{Y}_{N}$.

The Optimal Hybrid Smoothing Problem for Linear Discrete-Time Systems:

Find the pair $\left(\hat{r}_{s}(k), \hat{x}_{s}^{\left(\hat{r}_{s}(k)\right)}(k)\right), k \in[0, N]$ minimizing the performance index (6) based on the whole observed information $\mathcal{Y}_{N}$.

Remark 2.1 In the cases that we want to estimate the mode distributions, i.e., the stochastic properties of active modes, not each mode at each time, it often happen that we can obtain better estimation performances by fixed-time interval smoothing rather than filtering because, in the cases of filtering, as the system-driven time (the observation time) passes, the switchings between modes tend to arise and so we can feel difficulties on that we decide over which time interval we can obtain good estimation. As such example, we can consider the problem to estimate a distribution of active mode (for example, fine mode, cloudy mode and rainy mode) on the solar systems, the case that we want to obtain information of distributions of active agents on multiagents systems (for example, rescue robot systems) over long run time. This is the main reason we consider the fixed-time interval smoothing problem in this paer.

\section{Principles of Hybrid Optimality}

In the previous section we have formulated the estimation problems of the system states and mode distributions for the linear discrete-time hybrid systems, and we have shown the optimalities of the values of the performance indices for the pair of the optimal state and optimal mode distribution minimizing the value of the performance indices at each time $k$. In this section we show that the principles of optimality, which give theoretical bases for constructing estimation algorithms studied in the following section, hold over each time interval for the optimal control by regarding the system state and candidate of mode distributions as a pair of state. 
With regard to the optimal control problems considered in this paper, it is obvious that principle of optimality does not hold for the optimal trajectory $x^{*}(\cdot)$ with optimal control input $w_{d,(\cdot)}^{(r) *}(\cdot)$ and each performance index for each mode distribution candidate $r \in \mathcal{N}_{0}$. However, for the pair $\left(\hat{r}_{(\cdot)}(\cdot), x(\cdot)\right)$ of the optimal mode distribution candidate and optimal trajectory with the optimal control inputs $w_{d,(\cdot)}^{(r) *}(\cdot)$, the following principles of hybrid optimality hold.

Consider the following system

$$
\begin{aligned}
& x(k+1)={\overline{A_{d}}}^{(r)}(k) x(k)+w_{d}(k) \\
& y(k)=H_{d}(k, \theta(k)) x(k)+v_{d}(k, \theta(k))
\end{aligned}
$$

and the performance indices (4)-(6).

We consider the following three optimal control problems for the system (7) and the performance indices (4)-(6):

\section{Problem (A): Forward Optimal Control Prob- lem}

Consider the system ( 7$)$ with the initial state $x(0)$. Find the pair $\left(\hat{r}_{f}(l), w_{d, f}^{\left(\hat{r}_{f}(l)\right) *}(l)\right), l \in[0, k]$ minimizing the value of the performance index (4) based on the causal part $\mathcal{Y}_{k}=\{y(l) \mid 0 \leq l \leq k\}$ of the observed information $\mathcal{Y}_{N}$.

\section{Problem (B): Backward Optimal Control Prob-} lem

Consider the system (7) with the initial state $x(k)$. Find the pair $\left(\hat{r}_{b}(l), w_{d, b}^{\left(\hat{r}_{b}(l)\right) *}(l)\right), l \in[k, N]$ minimizing the value of the performance index (5) based on the anti-causal part $\overline{\mathcal{Y}}_{k}=\{y(l) \mid k \leq l \leq N\}$ of the observed information $\mathcal{Y}_{N}$.

\section{Problem (C): Fixed-Interval Optimal Control}

\section{Problem}

Consider the system (7) with the initial state $x(0)$. Find the pair $\left(\hat{r}_{s}(k), w_{d, s}^{\left(\hat{r}_{s}(k)\right) *}(k)\right), k \in[0, N]$ minimizing the value of the performance index (6) based on the whole observed information $\mathcal{Y}_{N}$.

Proposition A (Principle of Hybrid Optimality (A)) Consider the optimal control problem (A) on the time interval $[0, k]$. Also consider the optimal control problem minimizing the performance index

$$
\begin{aligned}
& J_{f}^{(r)}\left(\tau, x, w_{d}(\cdot)\right) \\
&= \sum_{l=0}^{\tau-1} L^{(r)}\left(l, x(l), w_{d}(l), y(l)\right)+\Phi_{0}(x(0)), \\
& 1<\tau<k
\end{aligned}
$$

for the system (7) with the initial state $x(0)$ on the partial time interval $[0, \tau]$ and then let the pair of optimal control inputs be $\left(\hat{\hat{r}}_{f}(l), w_{d, f}^{\left(\hat{\hat{r}}_{f}(l)\right) * *}(l)\right), l \in[0, \tau]$. Then $\left(\hat{r}_{f}(l), w_{d, f}^{\left(\hat{r}_{f}(l)\right) *}(l)\right)=\left(\hat{\hat{r}}_{f}(l), w_{d, f}^{\left(\hat{\hat{r}}_{f}(l)\right) * *}(l)\right), l \in[0, \tau]$ holds.

Proposition B (Principle of Hybrid Optimality (B)) Consider the optimal control problem (B) on the time interval $[k, N]$. Also consider the optimal control problem minimizing the performance index

$$
\begin{aligned}
& J_{b}^{(r)}\left(\tau, x, w_{d}(\cdot)\right) \\
= & \sum_{l=\tau}^{N-1} L^{(r)}\left(l, x(l), w_{d}(l), y(l)\right)+\Phi_{N}(x(N)), \\
& k<\tau<N-1
\end{aligned}
$$

for the system (7) with the initial state $x(\tau)$ on the partial time interval $[\tau, N]$ and then let the pair of optimal control inputs be $\left(\hat{\hat{r}}_{b}(l), w_{d, b}^{\left(\hat{\hat{r}}_{b}(l)\right) * *}(l)\right), l \in[\tau, N]$. Then $\left(\hat{r}_{b}(l), w_{d, b}^{\left(\hat{r}_{b}(l)\right) *}(l)\right)=\left(\hat{\hat{r}}_{b}(l), w_{d, b}^{\left(\hat{\hat{r}}_{b}(l)\right) * *}(l)\right), \quad l \in[\tau, N]$ holds.

Theorem C (Principle of Hybrid Optimality (C)) Consider the optimal control problem (C) on the fixed time interval $[0, N]$. Split the performance index (6) into the two parts (4) and (5) and also consider the optimal control problems (A) on $[0, k]$ and (B) on $[k, N]$ for the system $(7)$ with initial state $x(0)$ and $x(k)$ respectively. Then at each time $k\left(\hat{r}_{f}(l), w_{d, f}^{\left(\hat{r}_{f}(l)\right) *}(l)\right)$, $l \in[0, k]$ and $\left(\hat{r}_{b}(l), w_{d, b}^{\left(\hat{r}_{b}(l)\right) *}(l)\right), l \in[k, N]$ are optimal input minimizing the values of the (4) and (5) to be used in order to compose the solution of the fixed-interval optimal control problem (C), i.e., at any time $k \in[0, N]$ $\left(\hat{r}_{s}(l), w_{d, s}^{\left(\hat{r}_{f}(l)\right) *}(l)\right)=\left(\hat{r}_{f}(l), w_{d, f}^{\left(\hat{r}_{f}(l)\right) *}(l)\right), l \in[0, k]$ and $\left(\hat{r}_{s}(l), w_{d, s}^{\left(\hat{r}_{f}(l)\right) *}(l)\right)=\left(\hat{r}_{b}(l), w_{d, b}^{\left(\hat{r}_{b}(l)\right) *}(l)\right), l \in[k, N]$ hold.

In this appendix we give only a proof of Proposition

A. The others can be shown by the similar arguments.

(Proof of Proposition A) We split the pair $\left(\hat{r}_{f}(l), w_{d, f}^{\left(\hat{r}_{f}(l)\right) *}(l)\right), l \in[0, k]$ of the optimal control inputs into the following two parts:

$$
\begin{aligned}
& \left(\hat{r}_{f, 1}, w_{d, f, 1}^{\left(\hat{r}_{f, 1}\right) *}\right)=\left(\hat{r}_{f}(l), w_{d, f}^{\left(\hat{r}_{f}(l)\right) *}(l)\right) l \in[0, \tau] \\
& \left(\hat{r}_{f, 2}, w_{d, f, 2}^{\left(\hat{r}_{f, 2}\right) *}\right)=\left(\hat{r}_{f}(l), w_{d, f}^{\left(\hat{r}_{f}(l)\right) *}(l)\right) l \in[\tau+1, k]
\end{aligned}
$$

Now we assume

$$
\left(\hat{r}_{f, 1}, w_{d, f, 1}^{\left(\hat{r}_{f, 1}\right) *}\right) \neq\left(\hat{\hat{r}}_{f}, w_{d, f}^{\left(\hat{\hat{r}}_{f}\right) * *}\right) \text { on }[0, \tau] .
$$

Then there exists the pair of control inputs $\left(\hat{\hat{r}}_{f}, w_{d, f}^{\left(\hat{\hat{r}}_{f}\right) * *}\right)$ giving less value of the performance index (8) than $\left(\hat{r}_{f, 1}, w_{d, f, 1}^{\left(\hat{r}_{f, 1}\right) *}\right)$ and so the pair of control inputs consisting of $\left(\hat{\hat{r}}_{f}, w_{d, f}^{\left(\hat{\hat{r}}_{f}\right) * *}\right)$ and $\left(\hat{r}_{f, 2}, w_{d, f, 2}^{\left(\hat{r}_{f, 2}\right) *}\right)$ gives less value of the performance index $(4)$ than $\left(\hat{r}_{f}(l), w_{d, f}^{\left(\hat{r}_{f}(l)\right) *}(l)\right)$, $l \in[0, k]$. This contradicts with the optimality of $\left(\hat{r}_{f}(l), w_{d, f}^{\left(\hat{r}_{f}(l)\right) *}(l)\right), \quad l \in[0, k]$. Therefore $\left(\hat{r}_{f}(l), w_{d, f}^{\left(\hat{r}_{f}(l)\right) *}(l)\right)=\left(\hat{\hat{r}}_{f}(l), w_{d, f}^{\left(\hat{\hat{r}}_{f}(l)\right) * *}(l)\right), \quad l \in[0, \tau]$ holds. (Q.E.D.)

\section{Hybrid Estimation Algorithms}

In the previous section we have studied the principles of hybrid optimality, which give the theoretical bases 
for the construction of the hybrid estimation algorithms for the system states and mode distributions. In this section we consider actual methods of the construction of the estimation algorithms.

Define

$$
\begin{gathered}
V_{f}(k):=V_{f}^{\left(\hat{r}_{f}(k)\right)}(k), V_{b}(k):=V_{b}^{\left(\hat{r}_{b}(k)\right)}(k), \\
V_{s}(k):=V_{s}^{\left(\hat{r}_{s}(k)\right)}(k) .
\end{gathered}
$$

In order to construct the hybrid estimation algorithms, which give solutions of the hybrid estimation problems for the system states and mode distributions formalized in Section 2, in the filtering case, we need to first obtain the values of $\hat{x}_{f}^{(r)}(k), V_{f}^{(r)}(k)$ and $\hat{r}_{f}(k)$ and then obtain the value of $V_{f}(k)$ at each time $t$ using them. In the smoothing case, we need to additionally obtain the values of $\hat{x}_{b}^{(r)}(k), V_{b}^{(r)}(k)$ and $\hat{r}_{b}(k)$ and finally obtain the value of $V_{s}(k)$ at each time $k$ using the values of $\hat{x}_{s}^{(r)}(k), V_{s}^{(r)}(k)$ and $\hat{r}_{s}(k)$.

We assume the following condition:

A1: $\quad$ The matrices ${\overline{A_{d}}}^{(r)}(k), k=0,1, \cdots$ are invertible.

\subsection{Optimal Hybrid Filtering}

Since the value function $V_{f}(k)$, which is needed in order to construct the forward optimal filtering algorithm, can be written as

$$
V_{f}(k)=\min _{r \in \mathcal{N}_{0}}\left\{\min _{x(\cdot) \in \mathbf{R}^{\mathbf{n}}}\left\{\inf _{w_{d}(\cdot) \in \mathbf{R}^{\mathbf{n}}} J_{f}^{(r)}\left(k, x(\cdot), w_{d}(\cdot)\right)\right\}\right\},
$$

we can obtain the value of $V_{f}(k)$ by applying the dynamic programming method to the system (1) and the performance index (4) for each $x$ and $r$, deriving the Hamilton-Jacobi-Bellman (HJB) equation for each $x$ and $r$ and solving the HJB equation with an initial condition.

The dynamic programming (DP) equations associated with the forward control problem to minimize $J_{f}^{(r)}$ with regard to $w_{d}(\cdot)$ are given as follows:

$$
\begin{aligned}
& V_{f}^{(r)}(k+1, x) \\
= & \min _{w_{d}}\left\{L^{(r)}\left(k,{\overline{A_{d}}}^{(r),-1}(k)\left(x-w_{d}\right), w_{d}, y(k)\right)\right. \\
& \left.+V_{f}^{(r)}(k, x)\right\}, \\
& V_{f}^{(r)}(0, x)=\Phi_{0}(x), r \in \mathcal{N}_{0}
\end{aligned}
$$

Let

$$
V_{f}^{(r)}(k, x)=x^{\prime} K_{f}^{(r)}(k) x+2\left(p_{f}^{(r)}(k)\right)^{\prime} x+q_{f}^{(r)}(k)(10)
$$

for some functions $K_{f}^{(r)}, p_{f}^{(r)}$ and $q_{f}^{(r)}$ with appropriate dimensions. Then we obtain the following minimizing $w_{d}(\cdot)$.

$$
\begin{aligned}
& w_{d, f}^{(r) *}(k, x) \\
= & x-{\overline{A_{d}}}^{(r)}(k) S_{d}^{(r)}(k)\left({\overline{A_{d}^{\prime} M_{d}}}^{(r)}(k) x\right. \\
& \left.+{\overline{H_{d}^{\prime} N_{d}}}^{(r)}(k) y(k)-p_{f}^{(r)}(k)\right)
\end{aligned}
$$

where

$$
S_{d}^{(r)}(k)=\left[{\overline{A_{d}^{\prime} M_{d} A_{d}}}^{(r)}(k)+{\overline{H_{d}^{\prime} N_{d} H_{d}}}+K_{f}^{(r)}(k)\right]^{-1} .
$$

Then we obtain the following matrix difference equations, forward vector equations and scalar equations with initial conditions:

$$
\begin{aligned}
& K_{f}^{(r)}(k+1)={\overline{M_{d}}}^{(r)}(k)-{\overline{M_{d} A_{d}}}^{(r)}(k) \\
& \times S_{d}^{(r)}(k){\overline{A_{d}^{\prime} M_{d}}}^{(r)}(k), K_{f}^{(r)}(0)=D_{0}(11) \\
& p_{f}^{(r)}(k+1)=-{\overline{M_{d} A_{d}}}^{(r)}(k) S_{d}^{(r)} \\
& \quad \times\left[{\overline{H_{d}^{\prime} N_{d}}}^{(r)}(k) y(k)-p_{f}^{(r)}(k)\right], p_{f}^{(r)}(0)=-D_{0} \hat{x}_{0}(12) \\
& q_{f}^{(r)}(k+1) \\
& =-\left[{\overline{H_{d}^{\prime} N_{d}}}^{(r)}(k) y(k)-p_{f}^{(r)}(k)\right]^{\prime} S_{d}^{(r)}(k) \\
& \quad \times\left[{\overline{H_{d}^{\prime} N_{d}}}^{(r)}(k) y(k)-p_{f}^{(r)}(k)\right] \\
& +y^{\prime}(k){\overline{N_{d}}}^{(r)}(k) y(k)+q_{f}^{(r)}(k), q_{f}^{(r)}(0)=\hat{x}_{0}^{\prime} D_{0} \hat{x}_{0}(13)
\end{aligned}
$$

For any given $k$, by letting $\partial V_{f}^{(r)} / \partial x=0$, we obtain

$$
K_{f}^{(r)}(k) x+p_{f}^{(r)}(k)=0 .
$$

Since it can be shown that the matrix $K_{f}^{(r)}(k)$ is positive-definite, we obtain

$$
\hat{x}_{f}^{(r)}(k)=-\left(K_{f}^{(r)}(k)\right)^{-1} p_{f}^{(r)}(k)
$$

as the minimizer of $V_{f}^{(r)}(k, x)$. Then we obtain

$$
\begin{gathered}
\hat{x}_{f}^{(r)}(k+1)=-\left(K_{f}^{(r)}(k+1)\right)^{-1} p_{f}^{(r)}(k+1) \\
=\left[{\overline{M_{d}}}^{(r)}(k)-{\overline{M_{d} A_{d}}}^{(r)}(k) S_{d}^{(r)}(k){\overline{A_{d}^{\prime} M_{d}}}^{(r)}(k)\right]^{-1} \\
\times{\overline{M_{d} A_{d}}}^{(r)}(k) S_{d}^{(r)} \\
\times\left[{\overline{H_{d}^{\prime} N_{d}}}^{(r)}(k) y(k)+K_{f}^{(r)}(k) \hat{x}_{f}^{(r)}(k)\right], \hat{x}_{f}^{(r)}(0)=\hat{x}_{0}(14)
\end{gathered}
$$

and

$$
\begin{aligned}
& q_{f}^{(r)}(k+1) \\
=- & {\left[{\overline{H_{d}^{\prime} N_{d}}}^{(r)}(k) y(k)+K_{f}^{(r)}(k) \hat{x}_{f}^{(r)}(k)\right]^{\prime} S_{d}^{(r)}(k) } \\
& \times\left[{\overline{H_{d}^{\prime} N_{d}}}^{(r)}(k) y(k)+K_{f}^{(r)}(k) \hat{x}_{f}^{(r)}(k)\right] \\
+ & y^{\prime}(k){\overline{N_{d}}}^{(r)}(k) y(k)+q_{f}^{(r)}(k), q_{f}^{(r)}(0)=\hat{x}_{0}^{\prime} D_{0} \hat{x}_{0} .
\end{aligned}
$$

We also obtain

$$
V_{f}^{(r)}(k)=-\left(\hat{x}_{f}^{(r)}(k)\right)^{\prime} K_{f}^{(r)}(k) \hat{x}_{f}^{(r)}(k)+q_{f}^{(r)}(k) .
$$

Now we have the following filtering algorithm, which gives the solution of the Optimal Hybrid Filtering Problem for Linear Continuous-Time Systems. 
*** Optimal hybrid filtering algorithm***

Step 1) Obtain $K_{f}^{(r)}(k), \hat{x}_{f}^{(r)}(k)$ and $q_{f}^{(r)}(k)$ for $r \in \mathcal{N}_{0}$ and $k \in[0, N]$ by solving (11), (14) and (15) with initial conditions.

Step 2) Choose $\hat{r}_{f}(k)$ that minimizes

$$
V_{f}^{(r)}(k)=-\left(\hat{x}_{f}^{(r)}(k)\right)^{\prime} K_{f}^{(r)}(k) \hat{x}_{f}^{(r)}(k)+q_{f}^{(r)}(k) .
$$

Then the most probable distribution is $\phi^{\left(\hat{r}_{f}(k)\right)}(\cdot)$ and the optimal filter is given by

$$
\left(\hat{r}_{f}(k), \hat{x}_{f}(k)\right)=\left(\hat{r}_{f}(k), \hat{x}_{f}^{\left(\hat{r}_{f}(k)\right)}(k)\right) .
$$

\subsection{Optimal Hybrid Smoothing}

Next we need to obtain the value of $V_{b}(k)$ at each time $k$ to construct the optimal hybrid smoothing algorithm. Since the value function $V_{b}(k)$ can be written as

$$
V_{b}(k)=\min _{r \in \mathcal{N}_{0}}\left\{\min _{x(\cdot) \in \mathbf{R}^{\mathbf{n}}}\left\{\inf _{w_{d}(\cdot) \in \mathbf{R}^{\mathbf{n}}} J_{b}^{(r)}\left(k, x(\cdot), w_{d}(\cdot)\right)\right\}\right\},
$$

we can obtain the value of $V_{b}(k)$ by applying the dynamic programming method to the system (1) and the performance index (5) for each $x$ and $r$, deriving the Hamilton-Jacobi-Bellman (HJB) equation for each $x$ and $r$ and solving the HJB equation with a terminal condition.

The dynamic programming (DP) equations associated with the backward control problem to minimize $J_{b}^{(r)}$ with regard to $w_{d}(\cdot)$ are given as follows:

$$
\begin{aligned}
& V_{b}^{(r)}(k, x) \\
= & \min _{w_{d}}\left\{L^{(r)}\left(k, x, w_{d}, y(k)\right)\right. \\
& \left.+V_{b}^{(r)}\left(k+1, \bar{A}_{d}^{(r)}(k) x+w_{d}\right)\right\}, \\
& V_{b}^{(r)}(N, x)=\Phi_{N}(x), r \in \mathcal{N}_{0}
\end{aligned}
$$

Let

$$
V_{b}^{(r)}(k, x)=x^{\prime} K_{b}^{(r)}(k) x+2\left(p_{b}^{(r)}(k)\right)^{\prime} x+q_{b}^{(r)}(k)(16)
$$

for some functions $K_{b}^{(r)}, p_{b}^{(r)}$ and $q_{b}^{(r)}$ with appropriate dimensions. Then we obtain the following minimizing $w_{d}(\cdot)$.

$$
\begin{aligned}
w_{b}^{(r) *}(k, x)=\left\{-{\overline{A_{d}}}^{(r)}(k)+T_{d}^{(r)}(k) \overline{M_{d} A_{d}}(k)\right\} x(k) \\
-T_{d}^{(r)}(k) p_{f}^{(r)}(k+1)
\end{aligned}
$$

where

$$
T_{d}^{(r)}(k)=\left[{\overline{M_{d}}}^{(r)}(k)+K_{b}^{(r)}(k+1)\right]^{-1} .
$$

Then we obtain the following matrix difference equations, backward vector equations and scalar equations with terminal conditions:

$$
\begin{array}{r}
K_{b}^{(r)}(k)={\overline{A_{d}^{\prime} M_{d} A_{d}}}^{(r)}(k)-{\overline{A_{d}^{\prime} M_{d}}}^{(r)}(k) \\
\times T_{d}^{(r)}(k){\overline{M_{d} A_{d}}}^{(r)}(k)+{\overline{H_{d}^{\prime} N_{d} H_{d}}}^{(r)}(k), \\
K_{b}^{(r)}(N)=D_{N}
\end{array}
$$

$$
\begin{aligned}
p_{b}^{(r)}(k) & =\overline{A_{d}^{\prime} M_{d}}(k) T_{d}^{(r)}(k) p_{b}^{(r)}(k+1) \\
& -{\overline{H_{d}^{\prime} N_{d}}}^{(r)}(k) y(k), p_{b}^{(r)}(N)=-D_{N} \hat{x}_{N}
\end{aligned}
$$

$$
\begin{aligned}
& q_{b}^{(r)}(k) \\
=- & p_{b}^{(r)^{\prime}}(k+1) T_{d}^{(r)}(k) p_{b}^{(r)}(k+1)+q_{b}^{(r)}(k+1) \\
& +y^{\prime}(k) \overline{N_{d}}(k+1) y(k), q_{b}^{(r)}(N)=\hat{x}_{N}^{\prime} D_{N} \hat{x}_{N}
\end{aligned}
$$

For any given $k$, by letting $\partial V_{b}^{(r)} / \partial x=0$, we obtain

$$
K_{b}^{(r)}(k) x+p_{b}^{(r)}(k)=0
$$

Since it can be also shown that the matrix $K_{b}^{(r)}(k)$ is positive-definite, we obtain

$$
\hat{x}_{b}^{(r)}(k)=-\left(K_{b}^{(r)}(k)\right)^{-1} p_{b}^{(r)}(k)
$$

as the minimizer of $V_{b}^{(r)}(k, x)$. Then we obtain

$$
\begin{aligned}
& \hat{x}_{b}^{(r)}(k)=-\left(K_{b}^{(r)}(k)\right)^{-1} p_{b}^{(r)}(k) \\
&= {\left[{\overline{A_{d}^{\prime} M_{d} A_{d}}}^{(r)}(k)-{\overline{A_{d}^{\prime} M_{d}}}^{(r)}(k)\right.} \\
&\left.\times T_{d}^{(r)}(k){\overline{M_{d} A_{d}}}^{(r)}(k)+{\overline{H_{d}^{\prime} N_{d} H_{d}}}^{(r)}(k)\right]^{-1} \\
& \times\left[\overline{A_{d}^{\prime} M_{d}}(k) T_{d}^{(r)}(k) K_{b}^{(r)}(k+1) \hat{x}_{b}^{(r)}(k+1)\right. \\
&\left.+{\overline{H_{d}^{\prime} N_{d}}}^{(r)}(k) y(k)\right], \\
& \hat{x}_{b}^{(r)}(N)=\hat{x}_{N}
\end{aligned}
$$

and

$$
\begin{aligned}
& q_{b}^{(r)}(k) \\
=-\hat{x}_{b}^{(r)^{\prime}}(k+1) K_{b}^{(r)}(k+1) & T_{d}^{(r)}(k) \\
& \times K_{b}^{(r)}(k+1) \hat{x}_{b}^{(r)}(k+1) \\
+ & q_{b}^{(r)}(k+1)+y^{\prime}(k) \overline{N_{d}}(k+1) y(k), \\
q_{b}^{(r)}(N) & =\hat{x}_{N}^{\prime} D_{N} \hat{x}_{N} .
\end{aligned}
$$

We also obtain

$$
V_{b}^{(r)}(k)=-\left(\hat{x}_{b}^{(r)}(k)\right)^{\prime} K_{b}^{(r)}(k) \hat{x}_{b}^{(r)}(k)+q_{b}^{(r)}(k) .
$$

Using (10) and (16), we can express $V_{s}^{(r)}(k, x)$ as

$$
\begin{aligned}
& V_{s}^{(r)}(k, x)=x^{\prime}\left[K_{f}^{(r)}(k)+K_{b}^{(r)}(k)\right] x \\
&+2\left[p_{f}^{(r)}(k)+p_{b}^{(r)}(k)\right]^{\prime} x+q_{f}^{(r)}(k)+q_{b}^{(r)}(k)
\end{aligned}
$$

Let

$$
\partial V_{s}^{(r)} / \partial x=0
$$

and we obtain the following form.

$$
\hat{x}_{s}^{(r)}(k)=-\left[K_{f}^{(r)}(k)+K_{b}^{(r)}(k)\right]^{-1}\left(p_{f}^{(r)}(k)+p_{b}^{(r)}(k)\right)
$$

Since $p_{f}^{(r)}(k)=-K_{f}^{(r)}(k) \hat{x}_{f}^{(r)}(k)$ and $p_{b}^{(r)}(k)=$ $-K_{b}^{(r)}(k) \hat{x}_{b}^{(r)}(k)$, for each candidate $r$ of given distributions, we can obtain the following form of smoothed 
estimate at time $k$ by the forward and backward filtered estimates.

$$
\hat{x}_{s}^{(r)}(k)=K_{s}^{(r)}(k)\left[K_{f}^{(r)}(k) \hat{x}_{f}^{(r)}(k)+K_{b}^{(r)}(k) \hat{x}_{b}^{(r)}(k)\right]
$$

where $K_{s}^{(r)}(k)=\left[K_{f}^{(r)}(k)+K_{b}^{(r)}(k)\right]^{-1}$.

Now we have the following smoothing algorithm, which gives the solution of the Optimal Hybrid Smoothing Problem for Linear Discrete-Time Systems.

*** Optimal hybrid smoothing algorithm***

Step 1) Obtain $K_{b}^{(r)}(k), \hat{x}_{b}^{(r)}(k)$ and $q_{b}^{(r)}(k)$ for $r \in \mathcal{N}_{0}$ and $k \in[0, N]$ by solving (17), (20) and (21) with terminal conditions.

Step 2) Choose $\hat{r}_{s}(k)$ that minimizes

$$
V_{s}^{(r)}(k)=V_{f}^{(r)}(k)+V_{b}^{(r)}(k)
$$

where

$$
V_{b}^{(r)}(k)=-\hat{x}_{b}^{(r)^{\prime}}(k) K_{b}^{(r)}(k) \hat{x}_{b}^{(r)}(k)+q_{b}^{(r)}(k) .
$$

Then the most probable distribution is $\phi^{\left(\hat{r}_{s}(k)\right)}(\cdot)$ and the optimal smoother is given by

$$
\begin{aligned}
& \quad\left(\hat{r}_{s}(k), \hat{x}_{s}(k)\right)=\left(\hat{r}_{s}(k), \hat{x}_{s}^{\left(\hat{r}_{s}(k)\right)}(k)\right) \\
& =\left(\hat{r}_{s}(k), K_{s}^{\left(\hat{r}_{s}(k)\right)}(k)\left[K_{f}^{\left(\hat{r}_{s}(k)\right)}(k) \hat{x}_{f}^{\left(\hat{r}_{s}(k)\right)}(k)\right.\right. \\
& \left.\left.\quad+K_{b}^{\left(\hat{r}_{s}(k)\right)}(k) \hat{x}_{b}^{\left(\hat{r}_{s}(k)\right)}(k)\right]\right)
\end{aligned}
$$

where $K_{s}^{\left(\hat{r}_{s}(k)\right)}(k)=\left[K_{f}^{\left(\hat{r}_{s}(k)\right)}(k)+K_{b}^{\left(\hat{r}_{s}(k)\right)}(k)\right]^{-1}$.

\section{Numerical Examples}

In this section, we study numerical examples to demonstrate the effectiveness of the presented design algorithms.

We consider the following two mode systems and assume that the system parameters are as follows:

$$
\begin{gathered}
x(k+1)=A_{d}(k, \theta(k)) x(k)+w_{d}(k, \theta(k)), \\
x(0)=x_{0}, \theta(0)=i_{0} \\
y(k)=H_{d}(k, \theta(k)) x(k)+v_{d}(k, \theta(k))
\end{gathered}
$$

where

- Mode 1:

- Mode 2:

$A_{d}(\cdot, 1)=\left[\begin{array}{cc}0 & 1 \\ -0.8 & 0.6\end{array}\right], A_{d}(\cdot, 2)=\left[\begin{array}{cc}0.5 & 1 \\ -0.4 & 0.6\end{array}\right]$,

$$
H=[1,0]
$$

and

$$
M(t, i)=\left[\begin{array}{ll}
1 & 0 \\
0 & 1
\end{array}\right], N(t, i)=1, D_{0}=\left[\begin{array}{ll}
1 & 0 \\
0 & 1
\end{array}\right]
$$

for $i=1,2$. We set $\hat{x}_{0}=\operatorname{col}(-0.1,0)$ and the distribution of the initial mode $i_{0}$ as $(1 / 2,1 / 2) . w(\cdot, \cdot)$ and $v(\cdot, \cdot)$ are wide sense white noises which aren't restricted to be Gaussian white and the following properties:

$$
\begin{aligned}
& \mathbf{E}\left\{w_{d}(k, \theta(k))\right\}=0, \\
& \mathbf{E}\left\{w_{d}(k, \theta(k)) w_{d}^{\prime}(j, \theta(j))\right\}=0.1 \delta_{k j} I, \\
& \mathbf{E}\left\{v_{d}(k, \theta(k))\right\}=0, \\
& \mathbf{E}\left\{v_{d}(k, \theta(k)) v_{d}^{\prime}(j, \theta(j))\right\}=0.05 \delta_{k j} I
\end{aligned}
$$

The candidates of mode distributions are given as follows:

$$
\phi^{(1)}=\left(\frac{2}{5}, \frac{3}{5}\right), \phi^{(2)}=\left(\frac{1}{2}, \frac{1}{2}\right), \phi^{(3)}=\left(\frac{3}{5}, \frac{2}{5}\right)
$$

The paths of $\theta(k)$ are generated randomly, and the performances are compared under the same circumstance, that is, the same set of the paths so that the performances can be easily compared.

We consider the whole system (22) with the true mode distribution $\phi^{(3)}$ over the time interval $k \in[0,100]$. We verify the effectiveness of the presented hybrid estimation algorithms and compare the estimation performances for the optimal filtering and smoothing algorithms. In order to carry out these algorithms we solve the forward or backward triplet of the differential equations $(11)(14)(15)$ or $(17)(20)(21)$ with the initial or terminal conditions for given observation $y(\cdot)$ and each candidate $r=1,2,3$ of given distributions, and obtain the pair $\left(\hat{r}_{f}(k), \hat{x}_{f}(k)\right)$ minimizing $V_{f}^{(r)}(k)$ in the filtering case or the pair $\left(\hat{r}_{s}(k), \hat{x}_{s}(k)\right)$ minimizing $V_{s}^{(r)}(k)$ in the smoothing case for $k \in[0,100]$.

Fig. 1 and Fig. 2 show the square errors between the first components of the states and filtered values, and the states and smoothed values respectively. The mean square errors over the time interval $[0,100]$ are 0.0276 in the filtering case, and 0.0151 in the smoothing case respectively. From these figures and calculation results it is shown that the smoother gives better estimation than the filter. Filtered and smoothed mode distributions are given by Fig. 3 and Fig. 4. Notice that the vertical axes show the candidates of the mode distributions not the modes themselves. In Fig. 3 the filtered values of the mode distributions rapidly change to be left undecided. To the contrary in Fig. 4 the smoothed values of the mode distributions are firmly decided. Through these four figures it is shown that the optimal smoother presented in this paper gives better estimate perfromance than the optimal filter presented in the previous work $([24,25])$ from the point of view of both state and modes estimation.

\section{Concluding Remarks}

In this paper we have studied the state and mode estimation problems for linear discrete-time hybrid systems over the fixed time interval. The systems aren't restricted to the Markovian jump systems and the added noises aren't restricted to be Gaussian. With regard 


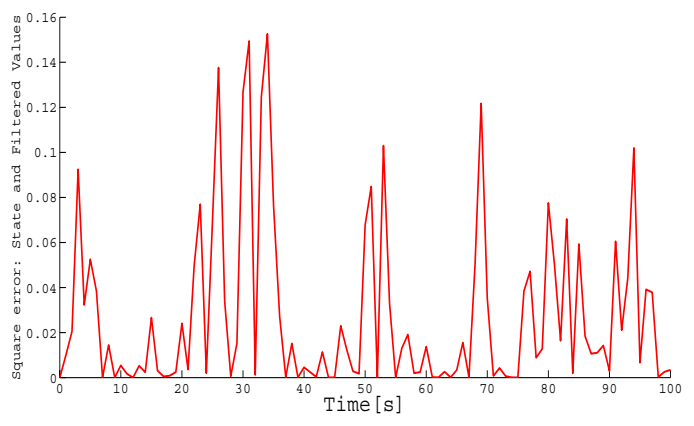

Fig. 1: The square errors between the state and filtered values

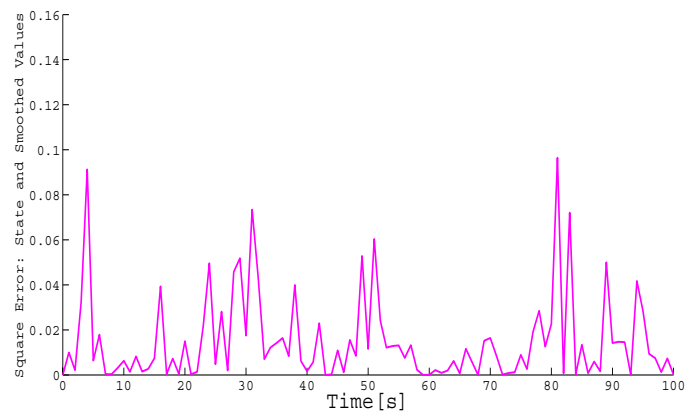

Fig. 2: The square errors between the state and smoothed values

to concrete examples of the systems considered in this chapter, refer to $[24,25]$. Those examples show that the systems and estimation algorithms presented in this chapter cover extreme broad classes of dynamical systems affected by the noises not to be restricted to be Gaussian. We have adopted the MPT approach. The state and mode estimation approach adopted in this paper guarantees the optimality of estimation performance in the meaning of MPT different from the previous work $([4,7])$.

In this paper we have considered the problems that both system state and modes are estimated. However we have considered the problems that the distributions of the modes over the fixed time interval not the modes themselves are estimated to grasp the global behavior of the hybrid systems over the long time intervals. In order to estimate both the system state and distributions of the modes we have introduced the averaged performance indices with respect to the candidates of the mode distributions for the averaged systems. This introduction of the averaged systems and performance indices prevents the computational complexity from increasing exponentially with time passage. For these performance indices we have formulated the optimal filtering and smoothing problems based on the avail-

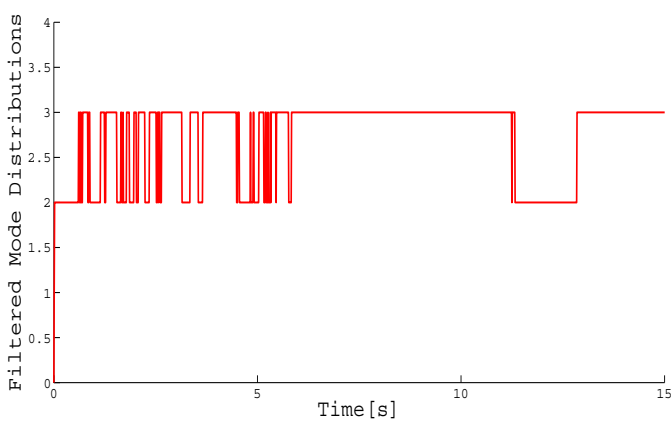

Fig. 3: The filtered mode distributions

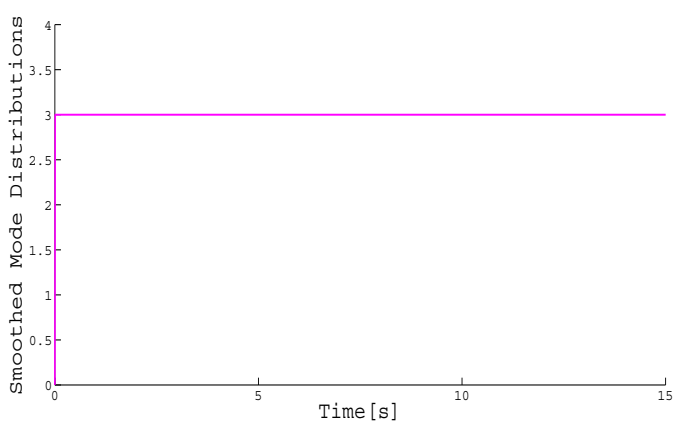

Fig. 4: The smoothed mode distributions

able observed information. The estimation problems have been reduced to the optimal control problems to find the noises minimizing the introduced performance indices. For these control problems we have shown that the principles of optimality hold. Based on the principles of optimality we have derived the forward and backward matrix difference equations and the forward and backward filter equations with the initial and terminal conditions respectively, which give the necessary conditions for the solvability of the optimal estimation problems. Then we have presented the optimal hybrid smoothing algorithm by the two filters approach. Finally we have studied the numerical examples to compare the estimation performances by filtering and smoothing. We have obtained the better estimation performance by the smoothing algorithm than the filtering algorithm from the point of view of both state and modes estimation.

With regard to continuous-time cases, refer to $[16,17$, 25]. In particular, in [17, 25], the cases that concerned systems are assumed to be Markovian jump systems is also considered. In these papers the concept of quasistationary distributions is introduced for the Markovian mode processes and near optimality of limiting estimators with the quasi-stationary distributions is shown. It is well known that the concept of quasi-stationary 
distribution is very important and highly practical to grasp behavior of stochastic processes over long run time. As a further research issue it is very significant that the quasi-stationary distributions of stochastic mode processes and estimator with these distributions are investigated for the discrete-time hybrid systems. Also notice that in this paper we have adopted the quadratic performance index for the noises not to be restricted to be Gaussian and so it can happen that the estimation does not always reflect the higher order moments of the distributions of the noises. In order to overcome this problem it will be hoped that we adopt some risk sensitive performance indices in future.

\section{References}

[1] T. Basar: Optimal Performance Levels for Minimax Filters, Predictors and Smoothers, Syst. Contr. Lett., Vol. 16, Issue 5, pp. 309-317, 1991.

[2] E. Blanco, P. Neveux and G. Thomas: The $\mathrm{H}_{\infty}$ Fixed-Interval Smoothing Problem for Continuous Systems, IEEE Trans. Signal Process., Vol. 54, No. 11, pp. 4085-4090, 2006.

[3] H. A. P. Blom and Y. Bar-Shalom: Time-reversion of a hybrid state stochastic difference system with a jump-linear smoothing application, IEEE Trans. Inform. Theory, Vol. 36, No. 4, pp. 836-847, 1990.

[4] O. L. V. Costa: Linear Minimum Mean Square Error Estimation for Discrete-Time Markovian Jump Linear Systems, IEEE Trans. Automat. Contr., Vol. 39, No. 8, pp.1685-1689, 1994.

[5] O. L. V. Costa, M. D. Fragoso and R. P. Marques: Discrete-Time Markov Jump Linear Systems, Springer, London, 2005.

[6] H. Cox: Estimation of State Variables via Dynamic Programming, Proc. 1964 Joint Automatic Control Conf., Stanford, California, pp.376-381, 1964.

[7] M. D. Fragoso, O. L. V. Costa, J. Baczynski and N. Rocha: Optimal linear mean square filter for continuous-time jump linear systems, IEEE Trans. Automat. Contr., Vol. 50, No. 9, pp.1364-1369, 2005.

[8] D. C. Fraser and J. E. Potter: The Optimum Linear Smoother as a Combination of Two Optimum Linear Filters, IEEE Trans. Automat. Contr., Vol. AC-14, No. 4, pp.387-390, 1969.

[9] R. E. Helmick, W. D. Blair and S. A. Hoffman: Fixed-Interval Smoothing for Markovian Switching Systems, IEEE Trans. Inform. Theory, Vol. 41, No. 6, pp.1845-1855, 1995.
[10] A. H. Jazwinski: Filtering for Nonlinear Dynamical Systems, IEEE Trans. Automat. Contr., Vol. 11, pp.765-766, 1966.

[11] B. Lincoln and A. Rantzer: Relaxing Dynamic Programming, IEEE Trans. Automat. Contr., Vol. 51, No. 8, pp. 1249-1260, 2006.

[12] L. Ljung and T. Kailath: Backwards Markovian Models for Second-Order Stochastic Processes, IEEE Trans. Inform. Theory, Vol. 22, No. 4, pp.488-491, 1976.

[13] D. Q. Mayne: A Solution of the Smoothing Problem for Linear Dynamic Systems, Automatica, Vol. 4, No. 2, pp.73-92, 1966.

[14] J. S. Meditch: Orthogonal Projection and Discrete Optimal Linear Smoothing, J. SIAM Control, Vol. 5, No. 1, pp.74-89, 1967.

[15] G. Nakura: $H_{\infty}$ Estimation for Linear DiscreteTime Markovian Jump Systems by Game Theoretic Approach, Proceedings of the 42nd ISCIE International Symposium on Stochastic Systems Theory and Its Applications (SSS10), Okayama, Japan, pp.163-169, 2010.

[16] G. Nakura: An Approach to Noncausal Hybrid Estimation for Linear Continuous-Time Systems with Non-Gaussian Noises, Proceedings of SICE Annual Conference 2011, Tokyo, Japan, WeC1102, pp. 979-984, (CD-ROM) 2011.

[17] G. Nakura: An Approach to Hybrid Smoothing for Linear Continuous-Time Systems with NonGaussian Noises, Proceedings of the 43rd ISCIE International Symposium on Stochastic Systems Theory and Its Applications (SSS11), Shiga, Japan, pp. 63-72, FB2-2, (CD-ROM) 2011.

[18] H. E. Rauch: Solutions to the Linear Smoothing Problem, IEEE Trans., Automat., Contr., Vol. AC-8, pp.371-372, 1963.

[19] H. E. Rauch, F. Tung and C. T. Striebel: Maximum Likelihood Estimates of Linear Dynamic Systems, AIAA J., Vol. 3, No. 8, pp.1445-1450, 1965.

[20] G. S. Sidhu and U. B. Desai: New Smoothing Algorithms Based on Reversed-Time Lumped Models, IEEE Trans. Automat. Contr., Vol. 21, pp.538541, 1976.

[21] D. D. Sworder and R. O. Rogers: An LQG Solution to a Control Problem with Solar Thermal Receiver, IEEE Trans. Automat. Contr., Vol. 28, pp.971-978, 1983.

[22] G. Verghese and T. Kailath: A Further Note on Backwards Markovian Models, IEEE Trans. Inf. Theory, Vol. IT-25, No. 1, pp.121-124, 1979. 
[23] J. E. Wall, Jr., A. S. Willsky and N. R. Sandell, Jr.: On the Fixed-Interval Smoothing Problem, Stochastics, Vol. 5, pp.1-41, 1981.

[24] Q. Zhang: Optimal Filtering of Discrete-Time Hybrid Systems, J. Optim. Theory Appl., Vol. 100, No. 1, pp.123-144, 1999.

[25] Q. Zhang: Hybrid Filtering for Linear Systems with Non-Gaussian Disturbances, IEEE Trans. Automat. Contr., Vol. 45, pp.50-61, 2000. 